\title{
DESENVOLVIMENTO DE UMA APLICAÇÃO DE CONTROLE DE PRESENÇAS DE ACADÊMICOS COM USO DE RECONHECIMENTO ATRAVÉS DE BIOMETRIA
}

\author{
DEVELOPMENT OF AN APP TO CONTROL STUDENTS' ATTENDANCE BY USING BIOMETRIC \\ RECOGNITION
}

${ }^{1}$ Professor do Curso de Sistemas de Informação da Universidade Paranaense - UNIPAR.

${ }^{2}$ Professor do Curso de Sistemas de Informação da Universidade Paranaense - UNIPAR.

${ }^{3}$ Professor do Curso de Sistemas de Informação da Universidade Paranaense - UNIPAR.

${ }^{4}$ Professor do Curso de Sistemas de Informação da Universidade Paranaense - UNIPAR.

${ }^{5}$ Acadêmicos do Curso de Sistema de Informação da Universidade Paranaense - UNIPAR.
Recebido em maio de 2017 Aceito em julho de 2017
Carlos Marques ${ }^{1}$

Elyssandro Piffer ${ }^{2}$

Izabel Cristina Mioranza ${ }^{3}$

Leandro Clementino de Lima ${ }^{4}$

Arthur Pietchaki ${ }^{5}$

Carlos Eduardo Dutra Soares Rocha ${ }^{5}$

Eduardo Felipe Silva Ferro ${ }^{5}$

Gustavo Sabior Antoniassi ${ }^{5}$

Jordhan Emmanuel Marciano da Silva ${ }^{5}$

MARQUES, C.; PIFFER, E.; MIORANZA, I. C.; LIMA, L. C de. PIETCHAKI, A.; ROCHA, C. E. D. S.; FERRO, E. F. S.; ANTONIASSI, G. S.; SILVA, J. E. M. da. Desenvolvimento de uma aplicação de controle presenças de acadêmicos com uso de reconhecimento através de biometria. Akrópolis Umuarama, v. 25, n. 1, p. 81-88, jan./jun. 2017.

DOI: 10.25110/akropolis.v25i1.6677

Resumo: O processo de reconhecimento biométrico vem sendo aplicado há muito tempo, consistindo em uma alternativa para a identificação de pessoas, uma vez que apresenta características importantes para buscar o reconhecimento de padrões únicos que identificam os indivíduos. Dentre os reconhecimentos biométricos, a biometria digital, que utiliza como fonte de dados as impressões digitais, é a mais difundida, pois apresenta a facilidade da coleta das informações, bem como oferece uma boa margem de segurança quanto a identificação. Este tipo de reconhecimento possibilita a implementação de uma série de controles que são aplicados nos mais diversificados usos, sendo que para isto são utilizados equipamentos específicos para esta finalidade. No mercado existem vários tipos de equipamentos com este objetivo, porém como em qualquer equipamento, sempre vão existir necessidades que não são contempladas ou que são implementadas de forma diferente do que é exatamente o desejo do usuário, ou ainda, com uma série de funcionalidades que não serão utilizadas e acabam por onerar o equipamento, impactando diretamente no orçamento do projeto a ser implantado. Neste contexto, o presente trabalho busca demonstrar uma forma alternativa de implementação deste tipo de controle, utilizando o conceito de hardware livre através do Arduino, que possibilita a construção de projetos de equipamentos que, interligados com outros dispositivos, como o leitor de digitais, por exemplo, possam originar novos produtos com funcionalidades adequadas aos requisitos levantados. Além da parte física, o texto também apresenta a aplicação desenvolvida, que viabiliza a implementação de soluções para as necessidades apresentadas. A aplicação escolhida como forma de validação do processo foi algo que toma muito tempo dos professores em instituições de ensino, ou seja, o registro da presença dos alunos. Tarefa esta que demanda um tempo razoável que poderia estar 
sendo aplicado com mais propriedade no processo ensino-aprendizagem.

Palavras-Chave: Arduino; Biométrico; Digital; hardware livre e controle de presença; Impressão; Reconhecimento.

ABSTRACT: The biometric recognition process has been applied for a long time, consisting of an alternative for the identification of people, since it presents important characteristics seeking the recognition of unique traces to identify the individuals. Among the biometric recognitions, digital biometrics, which uses fingerprints as data source, is the most widespread, since its information is easily collected, as well as offering a good safety margin for identification. This type of recognition enables the implementation of a series of controls applied in the most diversified uses, using specific equipment for each purpose. There are several types of equipment available in the market for this purpose, but as in any piece of equipment, there will always be needs that have not been contemplated or that are implemented against the wishes of the user, or even a set of features that will not be used, but still burdening the equipment, with direct implications to the project's implementation budget. In this context, this work seeks to demonstrate an alternative form of implementation of such type of control, using the concept of free hardware through Arduino, which allows the construction of equipment projects that, by becoming interconnected with other devices, such as digital ones, for example, may result in new products with features that meet the requirements sought. In addition to the physical part, the paper will also presents the application that has been developed, which enables the implementation of solutions to meet the presented needs. The application chosen for validating the process was something that consumes quite some time from professors and teachers at educational institutions, that is, the registration of students' presence. This task demands a reasonable amount of time that could be better applied in the teaching-learning process.

KeYwords: Arduino; Biometrics; Digital; Free hardware; Presence control; Printing; Recognition.

\section{INTRODUÇÃO}

Em todas as atividades da humanidade o reconhecimento da identidade de uma pessoa é fundamental, pois é por meio dele que determinadas ações podem ser efetuadas ou não. Um porteiro de um edifício sempre recebe ordens para que não deixe pessoas estranhas adentrarem ao condomínio e, isto é feito pelo conhecimento e memorização da fisionomia que tem em relação aos moradores. Em outra situação, um banco só irá efetuar o pagamento de um che- que se a assinatura que consta no mesmo for identificada como válida se comparada com as registradas no cadastro da conta corrente. Em alguns casos o acesso a um determinado local só poderá ser efetuado se for digitada uma senha correta ou mediante do uso de crachás com identificação por tarjas magnéticas ou ainda por cartões com identificação por rádio frequência.

Apesar de práticos, estes métodos podem apresentar sérios problemas em relação ao nível de segurança, pois podem facilmente apresentar falhas. O porteiro pode enganar-se e deixar entrar alguém que não seja morador, principalmente quando estiver no início do trabalho e ainda não memorizou todos os moradores, ou ainda se o condomínio tiver um número elevado de moradores. Uma assinatura em um cheque pode ser falsificada facilmente por pessoas que tenham habilidade para tal e, cartões podem ser clonados.

Atualmente existem várias formas de reconhecimento de indivíduos, como voz, impressão digital, face, até mesmo a íris do olho, entre outras (MAGALHÃES, 2003). A identificação de um indivíduo pela voz se dá com base nas características fisiológicas e comportamentais da voz, para que o reconhecimento possa ser realizado com sucesso. Porém, o cadastramento das informações da voz pode ser um processo mais demorado se comparado a outros métodos. A apesar de ser um método mais caro que os demais, o reconhecimento da íris é muito seguro. Nesse procedimento são coletadas, por meio de infravermelho, informações desta membrana, gerando uma imagem comparativa com a de registro (CASTELANO, 2006). Para reconhecimentos de padrões faciais, é necessária a coleta de uma imagem em $3 \mathrm{D}$, que servirá de modelo para análise por meio de pontos específicos de referência.

A biometria digital utiliza as diferentes formas das linhas que as pessoas possuem nas pontas dos dedos (AQUINO, 2014). Essas linhas formam desenhos que possibilitam a identificação de cada indivíduo. Sendo que a biometria digital é a ferramenta de identificação pessoal mais difundida e utilizada entre as demais (PINHEIRO, 2008). O reconhecimento de digitais pode ser efetuado por um profissional especializado, que por meio de técnicas de visualização e comparação de pontos específicos, pode afirmar se uma digital é ou não de determinada pessoa. Este procedimento é demorado e custoso, 
pois exige a presença deste profissional. Ainda de acordo com Pinheiro, para solucionar esses problemas, foram criados os AFIS (Automatic Fingerprint Identification System ou Sistema Automatizado de Identificação de Impressões Digitais), que junto com o poder computacional atual, possibilitam agilidade de processamento em escala.

Um AFIS inicialmente apresenta o processo de cadastramento, o qual neste momento é coletada a impressão digital sob a supervisão de um administrador do sistema ou pessoa a quem ele delegue esta função. Os dados oriundos desta coleta serão atrelados ao cadastro da respectiva pessoa. Após essa etapa a pessoa cadastrada no sistema poderá ter acesso ao local (caso seja um sistema de restrição de acesso) por meio da comparação da digital presente no aparelho naquele momento com a obtida previamente na etapa de cadastramento, havendo compatibilidade, onde sua identificação será registrada, geralmente com informações adicionais como data e hora, para futuras consultas.

Em locais ou situações onde demanda-se um número elevado de reconhecimentos digitais, pode-se utilizar equipamentos específicos para esta finalidade. Dependendo das necessidades, uma instituição pode encontrar equipamentos que automatizam este processo, porém em casos específicos, os mesmos podem não atender a todos os requisitos propostos, ou ainda oferecer soluções para requisitos não presentes, que acaba por onerar demasiadamente o processo de implantação do sistema.

Em instituições de ensino, é comum a prática dos professores realizarem o registro de presença de acadêmicos. Uma forma de realizar estes registros é por meio de chamada, aluno por aluno, enquanto o professor verifica se o aluno chamado está presente. Também é comum que o profissional passe uma lista pela sala onde ocorre as aulas, em que cada aluno tem por sua responsabilidade assinar no local indicado confirmando sua presença naquele momento. Entretanto, há possibilidades de erros e até fraudes quanto a estes registros, como por exemplo, um aluno assinar o nome de outro, assim registrando uma falsa presença. Quando se utiliza do meio de chamada de aluno por aluno, existe uma perda de tempo relevante, tanto para o professor, quanto para os alunos, pois este tempo poderia ser utilizado em atividades bem mais proveitosas. Ao utilizar de um sistema de reconhecimento biométrico, busca-se minimizar estes problemas, pois o próprio aluno ao entrar na sala, registra sua presença, otimizando o tempo, já que o sistema registra o local, data e hora em que o mesmo efetuou o registro.

Buscando viabilizar o desenvolvimento e a implantação de um sistema de reconhecimento biométrico por impressão digital, propôs-se a implementação de um protótipo que envolva hardware e software e, que implemente um AFIS voltado ao controle de presenças de alunos em uma instituição de ensino superior. Utilizou-se assim uma plataforma de hardware livre de nome Arduino, que pode ser implementada a custos reduzidos, com a possibilidade de integração de vários componentes adicionais como módulos Ethernet para conexão em rede, leitor de impressões digitais, etc. Pela própria característica do projeto Arduino, existe uma facilidade relativamente grande em adicionar novas funcionalidades, estendendo assim a lista de requisitos que o mesmo pode atender. Quanto ao software, foi desenvolvido um sistema na linguagem $C$, para cadastrar e manter as informações dos acadêmicos e suas impressões digitais, bem como realizar o reconhecimento das digitais dos alunos e os respectivos registros de presença, possibilitando a geração dos relatórios necessários.

\section{IMPRESSÃO DIGITAL}

As impressões digitais são encontradas na derme, que é o tecido conjuntivo sobre o qual se apoia a epiderme, que é a porção superficial da pele (AQUINO, 2014). Na derme encontram-se as papilas, dispostas em uma série de linhas separadas por sulcos. A impressão digital de cada ser humano, se forma durante o sexto mês de gestação e durante o envelhecimento, apesar de ocorrer mudanças como tamanho, o formato permanece inalterado durante toda a vida do indivíduo (ABE, 2005 e Silva, 2006). Uma impressão digital é composta por três elementos: núcleo, deltas e linhas. As linhas são os elementos chave para a formação de uma digital. Através destes se formam todos os outros elementos. Deltas são figuras formadas pela junção de linhas de vários sentidos, fazendo parecer um triângulo. Os deltas possibilitam a divisão das impressões em diversas classes e regiões (marginal, nuclear, basilar). Já os núcleos são formados pelas linhas encontradas no centro de uma 
impressão digital (AQUINO, 2014).

Figura 1: Elementos Básicos das Impressões Digitais (PINHEIRO, 2008).

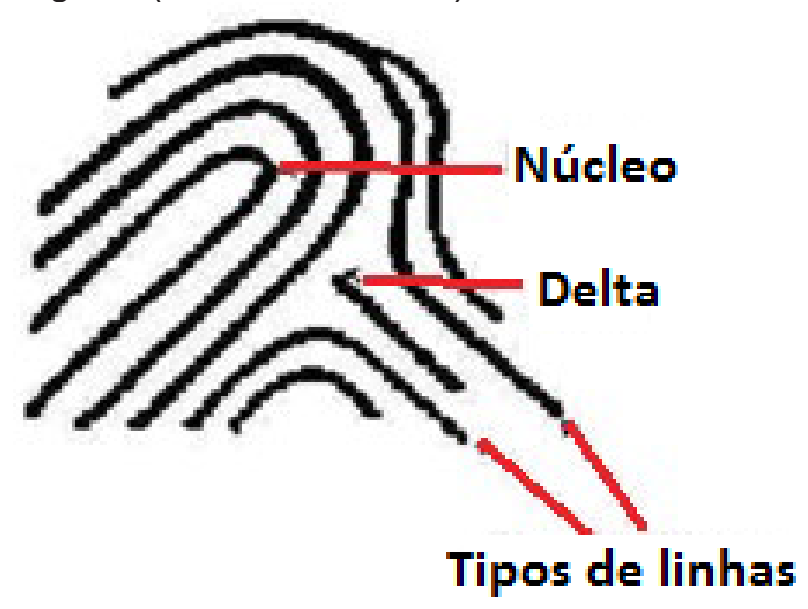

Antropólogos britânicos provaram cientificamente que as impressões digitais nunca mudam, apesar do decorrer do tempo e enveIhecimento da derme e epiderme, e nenhuma é exatamente igual a outra (MARANHÃO, 1989). As digitais são formadas por duas categorias: os elementos básicos e os elementos compostos. Os elementos básicos são os elementos que apresentam apenas uma linha, os compostos, por sua vez são formados por duas ou mais linhas, conforme apresentado na figura 2.

Figura 2: Elementos Básicos e Compostos (Costa, 2001)

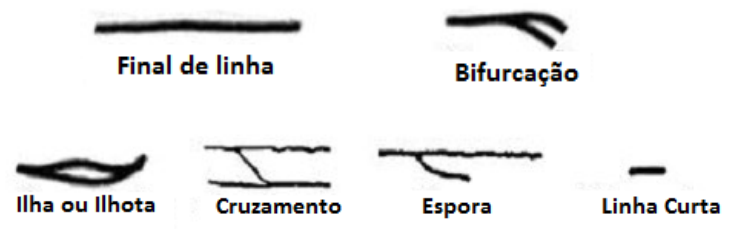

A partir dos dois elementos, temos a formação de uma digital, porém, não são apenas estes itens minúsculos que classificam uma identificação. Para que haja uma identificação, uma impressão digital completa, forma-se então, o conjunto das cristas e o delta, que correspondem ao maior ângulo ou a um triângulo isósceles entre as cristas (MAZI, 2009).

\section{SISTEMAS BIOMÉTRICOS}

Os sistemas biométricos tiveram início na década de 70 , devido à grande necessidade de agilizar o processamento de reconhecimento de características únicas em um indivíduo, esse ramo então teve um avanço, muitas pesquisas se iniciaram em cima dos dados recolhidos com tal tecnologia. Em 1902, a Comissão de Serviço de Nova York começou a utilizar as AFIS, logo em seguida, foi adotada em todo país. Algumas organizações como o FBI - Federal Bureau of Investigation que significa "Departamento Federal de Investigação" dos Estados Unidos, começaram a implantação de sistemas biométricos em locais restritos, algumas escolas passaram a usar o mesmo sistema em cantinas baseados na biometria da mão como forma de identificação. Também houve grande interesse do governo pela tecnologia de reconhecimento, principalmente pelos AFIS e seu baixo custo. (SINFIC, 2013, FORUMBIOMETRIA, 2013; ARAUJO, 2000). Sistemas biométricos, segundo Pinheiro (2008), podem ser definidos como um conjunto de hardware e software que realizam o reconhecimento de padrões com um propósito específico, que realizam o reconhecimento de características de um indivíduo, extraindo um modelo ou exemplo previamente armazenado em memória do hardware escolhido, usando as características de comparação.

Segundo Mazi (2009); Junior (2009), as impressões digitais se formam a partir das dobras de peles nos dedos, tanto nas mãos quanto nos pés. Também chamadas de ID - identity ou identidade, existem registros desde o período pré-histórico, mas foi em 1667, no período científico que comprovaram as características das ID's. Este estudo teve resultados positivos, e estes resultados foram chamados de Datiloscopia. O objetivo de um sistema biométrico é fornecer mecanismos para que seja possível, por meio das características adquiridas com a leitura da biometria do indivíduo, identificá-lo com um grau de certeza aceitável. O sistema de reconhecimento biométrico, consiste em um conjunto de hardware e software, utilizando-se destes para o reconhecimento de padrões, o qual opera através da aquisição de informação biométrica. Estes padrões anteriormente adquiridos virão a servir de modelo para comparação em uma segunda etapa, a do reconhecimento. Tal reconhecimento se dá na comparação da digital inserida, com a digital anteriormente modelada e armazenada em um banco de dados para que seja possível a identificação do indivíduo. Apesar de relativamente estável as características biomé- 
tricas, sofrem alterações com o passar do tempo, não se tem $100 \%$ de igualdade da digital do indivíduo, na maioria das vezes, com o mesmo equipamento que faz a coleta da amostra (PINHEIRO, 2008). Portanto, exige-se a necessidade de utilizar uma comparação não exata, mas com níveis de segurança que atestam percentualmente qual o grau aproximado que se deseja comparar a amostra previamente cadastrada com a digital captada em determinado momento.

\section{HARDWARE LIVRE E O ARDUINO}

Hardware Livre, assim como o movimento de Software Livre, propõe a implementação e distribuição de projetos de hardware de forma aberta, podendo ser replicado e adaptado livremente, estando disponíveis circuitos e drivers de comunicação com o mesmo.

O Arduino teve seu início na Itália, em 2005. Tinha como objetivo oferecer uma plataforma que permitisse uma fácil utilização e baixo custo, para a criação de projetos com componentes interativos (ARDUINO, 2016). Conforme Aquino (2014), a placa eletrônica Arduino consiste em um microcontrolador Atmel (que varia de acordo com a versão do Arduino), pinos digitais e analógicos de entrada e saída que permitem a conexão com outros componentes, entrada USB (que permite a conexão com outros dispositivos, como computadores) e conector de energia. O Arduino também possui um aspecto muito interessante como conexões com diversos componentes eletrônicos (sensores, botões, leds, etc.) e de módulos expansíveis, conhecidos como Shields, que nada mais são do que outras placas que possuem funcionalidades específicas (rede, wifi, bluetooth, servos, leitor de cartões SD - Secure Digital, etc.). Alguns modelos de Shields baseados em Arduino têm sido criados graças a plataforma Open Source do projeto, esses são denominados modelos não oficiais. São equipamentos desenvolvidos por empresas, alguns até mesmo pela própria comunidade. A comunidade está em toda parte no mundo, e estes estão listados na página oficial do projeto (ARDUINO, 2016).

Pode ter funcionalidades desenvolvidas em $\mathrm{C} / \mathrm{C}++$, sendo que utiliza uma IDE (Integrated Development Environment ou Ambiente de Desenvolvimento Integrado) escrita em Java. Após o programa ser compilado, é transmitido pela IDE para o dispositivo onde fica armazena- do em uma memória ROM (Read Only Memory). Portanto, uma vez programado, pode ser desligado que não irá "perder" o código.

\section{SOBRE O PROTÓTIPO DESENVOLVIDO}

Do ponto de vista do hardware, foi utilizado inicialmente um Arduino versão MEGA, pois apresenta um maior poder de processamento, bem com um maior espaço de armazenamento e uma quantidade maior de portas de comunicação. No Arduino fica o código compilado da aplicação desenvolvida, que é responsável pelo gerenciamento dos menus e o interfaceamento com os demais componentes; sendo assim a unidade central do processamento.

Outro dispositivo importante, e responsável pela aquisição, processamento e reconhecimento das impressões digitais é o leitor biométrico.

Como o modelo de leitor biométrico utilizado não apresenta um sensor para identificação do momento em que o usuário insere sua digital para leitura, houve a necessidade da implementação de um controle adicional através um sensor infra-vermelho. O objetivo deste controle é poupar o leitor de ficar em leitura contínua causando um desgaste desnecessário ao equipamento. Logo, somente quando o sensor infra-vermelho detecta a presença da digital, é enviado um sinal para o Arduino, que por sua vez aciona o leitor biométrico para efetuar a leitura.

O RTC - Real Time Clock é o módulo responsável por manter atualizada a data e hora do sistema, pois somente desta forma podemos garantir que, caso ocorra uma queda de energia e consequentemente desligue o equipamento, estas informações serão mantidas, não comprometendo assim o registro das passagens que vierem a acontecer após o reestabelecimento da energia para o módulo principal. Este módulo possui uma bateria que possibilita a continuidade de seu funcionamento durante o corte do fornecimento de energia externa.

Para que o usuário possa interagir meIhor com o equipamento, foi utilizado um display de LCD 16x2 (2 linhas de 16 caracteres cada) onde todas as mensagens e menus são mostrados ao mesmo.

Complementando uma interatividade melhor com o usuário, foram utilizados três botões: Up (cima), Select (seleciona) e Down (baixo). Esses botões serão utilizados pelo usuário 
para acessar os menus e efetuar as escolhas entre as opções disponibilizadas, possibilitando assim a sua operacionalização.

Também foi confeccionada uma caixa em MDF (Medium Density Fiberboard ou madeira de média densidade) com corte à laser, de forma a acondicinar melhor os componentes e facilitar o uso.

Figura 3: Vista frontal do protótipo

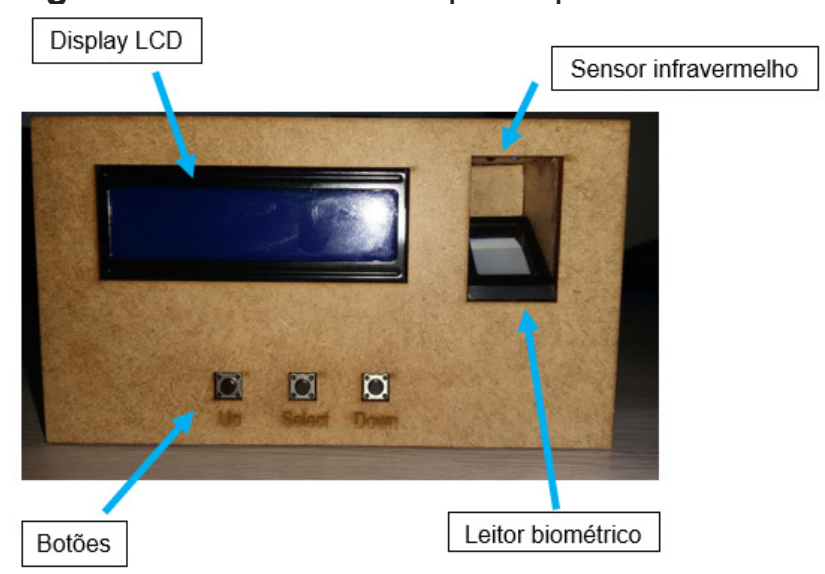

Figura 4: Vista interna do protótipo (A)

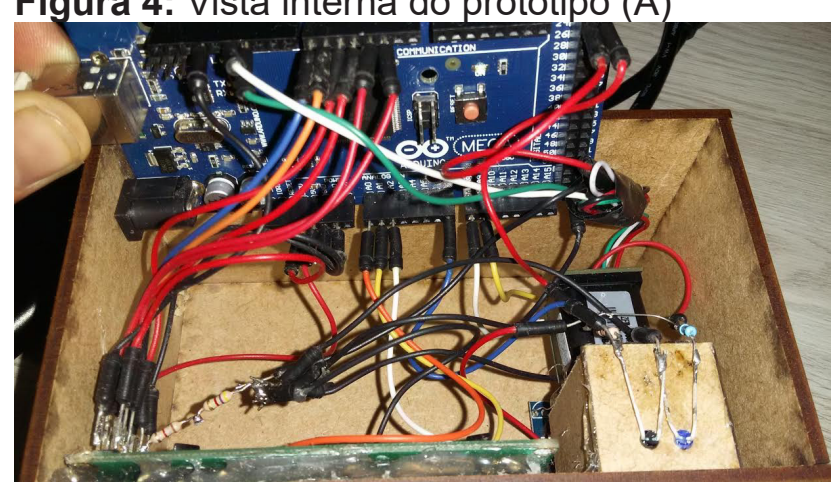

Figura 5: Vista interna do protótipo (B)

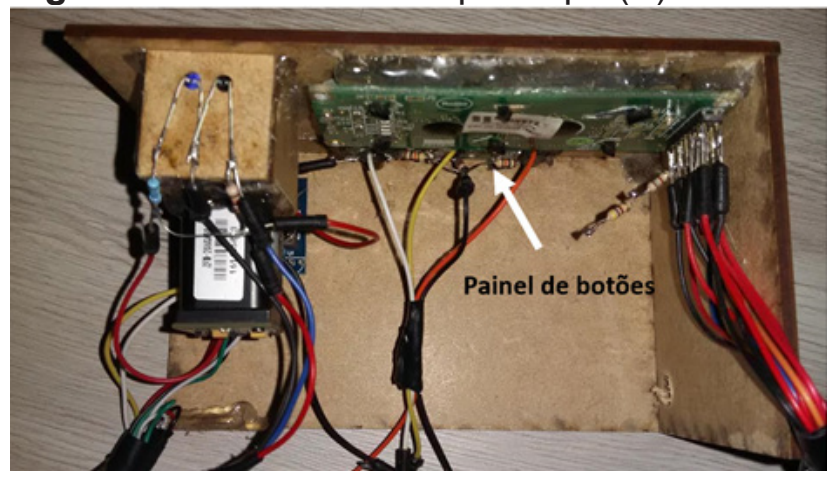

O protótipo tem como finalidade o registro das digitais e, posteriormente, o seu reconhecimento, com o respectivo registro das passagens constando os acadêmicos, as datas e horários. Também, existem funções adminis- trativas como o cadastramento e a exclusão das digitais, bem como a determinação de usuários com prerrogativa de administrador. Essas funções são acessadas por meio de menus que são mostrados na tela de LCD, porém só ficam disponíveis quando a digital de um administrador é reconhecida. Caso contrário, o software fica em estado de espera, aguardando uma digital a ser reconhecida, pois ele inicia pronto para o reconhecimento biométrico.

- A ordem dos passos na inicialização do equipamento é a seguinte:

- Ao ser inicializado, será mostrada uma mensagem de boas-vindas.

- Na sequência o Arduino tenta comunicar-se com o leitor biométrico até conseguir.

- Logo após é efetuada a leitura na memória do Arduino (EEPROM), buscando os dados sobre os usuários e administradores.

- Caso ainda não exista nenhum administrador cadastrado, será solicitada a sua inclusão, bem como sua impressão digital.

- Por fim, entra-se no modo de registro de passagens, aguardando por digitais a serem reconhecidas.

No modo de registro de passagens emite-se a mensagem para o usuário introduzir sua digital, além de mostrar a data e a hora atual. Esta rotina fica em execução e a cada segundo a data e a hora são atualizadas.

O Arduino executa o ciclo acima monitorando o sinal do sensor infravermelho que fica próximo ao leitor, na sua extremidade superior. Ao detectar a proximidade de qualquer objeto junto ao leitor, o Arduino envia o comando de aquisição da digital para o leitor, que tenta efetuar a leitura e a tentativa do reconhecimento da mesma. Caso a digital seja reconhecida como uma das digitais cadastradas no sistema, passa-se para a verificação se essa pertence a um usuário que esteja atribuído como administrador. Se o usuário da digital reconhecida for um administrador, um menu administrativo será mostrado no display para que se possa ter acesso às funcionalidades administrativas. Caso a digital reconhecida seja de usuário não administrador, a sua presença será registrada juntamente com a data e a hora atual. Quando a digital não é reconhecida é emitida uma mensagem de aviso.

Quando o software reconhece uma digi- 
tal administradora, mostra então um menu administrativo: Ao abrir o menu, são apresentadas as opções, sendo que a navegação entre os itens dos menus é efetuada através dos botões: up (acima), select (seleciona) e down (abaixo), que são monitorados pelo Arduino e dependendo das ações, as respostas são mostradas no display.

Em cada menu é apresentada uma seta $(\rightarrow)$ à esquerda do item selecionado, justamente para facilitar a identificação, por parte do usuário, de qual item está selecionado em determinado momento.

As funções do menu administrativo são:

- Identificar - Tenta identificar uma digital a ser capturada no leitor, informando o ID do usuário e o índice de confiabilidade. Caso não exista o reconhecimento, emite mensagem ao usuário.

- Cadastrar - Efetua o cadastramento do usuário comum.

- Cadastrar Administrador - Efetua o cadastramento de um administrador.

- Excluir - Permite a exclusão de um usuário através da seleção na lista de usuários.

- Sair - Volta para o registro de passagens.

Tabela 1: Custos do Protótipo

\begin{tabular}{lcc}
\hline \multicolumn{1}{c}{ Item } & Componentes novos & Utilizando sucata \\
\hline Arduino Mega & 80,00 & 80,00 \\
Módulo RTC & 20,00 & 20,00 \\
Caixa em MDF & 20,00 & 20,00 \\
Leitor de digitais & 246,00 & 246,00 \\
Display LCD 16x2 & 12,00 & \\
Sensor infravermelho & 8,00 & \\
3 botões & 3,00 & 366,00 \\
Total & 389,00 & \\
\hline
\end{tabular}

Um ponto negativo observado foi que pessoas com uma idade mais avançada ou que trabalham com produtos químicos, apresentam uma dificuldade maior para que o leitor consiga obter suas digitais.

De uma forma geral o protótipo superou as expectativas, sendo que se deseja implementar como trabalho futuro o armazenamento dos dados em cartão SD, a adição do Shield Ethernet, de forma a possibilitar o acesso ao dispositivo via rede, inclusive com a possibilidade de implementar um minisservidor Web para consulta aos dados online. Outra implementação futura, será o desenvolvimento de um sistema desktop para agilizar a manutenção do cadastro de usuários, bem como possibilitar a extração das informações, principalmente mediante de relatórios de controle e gerenciais.

\section{CONSIDERAÇÕES FINAIS}

A viabilidade da implementação de equipamentos utilizando o Arduino ficou evidenciada ao término dos trabalhos, uma vez que o comportamento do protótipo desenvolvido atendeu as expectativas. As possibilidades de adicionar novos componentes e, consequentemente novas funcionalidades também impressiona, abrindo oportunidades para que novos requisitos possam ser atendidos sem que haja a necessidade de demandar uma alteração estrutural muito grande.

Outro ponto positivo é a possibilidade de se alterar a programação do dispositivo, possibilitando uma maior adequação em relação às necessidades da instituição.

O custo relativamente baixo de aproximadamente $R \$ 389,00$ também é um fator que chama a atenção, ainda mais se considerado que parte dos componentes utilizados originaram-se de outros equipamentos em desuso. Como a instituição desenvolve um projeto, de arrecadação de lixo eletrônico, denominado Sistemas de Informação Solidário, o display de LCD, os botões e o sensor infravermelho, foram reaproveitados de outros dispositivos que iriam ser destinados à reciclagem, gerando assim um custo final de aproximadamente $\mathrm{R} \$ 366,00$.

\section{BIBLIOGRAFIA}

ABE, R. C. Dispositivos Biométricos com Comunicação USB. São Paulo. Faculdade de Engenharia de Sorocaba, 2005.

AQUINO, E. D. N. Sistemas automáticos de impressões digitais integrando Java e Arduino. Pernambuco: Editora Principia, 2014.

\section{ARAUJO, C. J. AFIS- Sistemas Automáticos} de Impressões Digitais. Brasília, 2000. Disponível em www.papiloscopistas.org/afis. html. Acesso em: 03 jun. 2016.

ARDUINO. Arduino. Disponível em: www. 
arduino.cc. Acesso 07 jun. 2016.

\section{BRENNER, G. P. S. Sistema de Controle de Acesso com Biometria da Digital. Santa Catarina: Editora PPGAU, 2011.}

CASTELANO. R. C. Introdução à Biometria. Santa Catarina: Universidade Federal de Santa Catarina, 2006. Santa Catarina: Universidade De São Paulo, 2006.

COSTA, L. R., OBELHEIRO, R. R., FRAGA, J. S. Introdução à Biometria. Santa Catarina: Universidade Federal de Santa Catarina, 2006.

MAGALHÃES. P. S., $4^{\text {a }}$ Conferência da Associação Portuguesa de Sistemas de Informação: Porto Portugal, 2003.

MARANHÃO, O. R. Curso Básico de Medicina Legal. 4. Ed. São Paulo: Editora: Revista dos Tribunais, 1989.

MAZI, R. C., Júnior. A. D. P. Identificação Biométrica Através da Impressão digital usando Redes Neurais Artificiais. Instituto tecnológico da Aeronáutica - ITA. São Paulo: Editora ENCITA, 2009.

\section{PINHEIRO, J. M. Biometria nos Sistemas} Computacionais - Você é a Senha. Rio de Janeiro: Editora Ciência Moderna, 2008.

SILVA, T. V. M. Estudo sobre Técnicas

de Pré-processamento e extração

de Características Utilizadas no

Reconhecimento de Impressões Digitais.

Monografia, Unipê, Paraíba, 2006.

\section{DESARROLLO DE UNA APLICACIÓN DE CONTROL DE PRESENCIAS DE ACADÉMICOS CON USO DE RECONOCIMIENTO A TRAVÉS DE BIOMETRÍA}

Resumen: El proceso de reconocimiento biométrico viene siendo aplicado desde hace mucho tiempo, consistiendo en una alternativa para la identificación de personas, ya que presenta características importantes para buscar el reconocimiento de estándares únicos que identifican a los individuos. Entre los reconocimientos biométricos, la biometría digital, que utiliza como fuente de datos las huellas dactilares, es la más difundida, pues presenta la facilidad de la recolección de las informaciones, así como ofrece un buen margen de seguridad en cuanto a la identificación. Este tipo de reconocimiento posibilita la imple- mentación de una serie de controles que se aplican en los más diversificados usos, siendo que para ello se utilizan equipos específicos para esta finalidad. En el mercado existen varios tipos de equipos con este objetivo, pero como en cualquier equipo, siempre van a existir necesidades que no son contempladas o que se implementan de forma diferente de lo que es exactamente el deseo del usuario, o aún, con una serie de funcionalidades que no serán utilizadas y terminan por cargar el equipo, impactando directamente en el presupuesto del proyecto a ser implantado. En este contexto, el presente trabajo busca demostrar una forma alternativa de implementación de este tipo de control, utilizando el concepto de hardware libre a través de Arduino, que posibilita la construcción de proyectos de equipos que, interconectados con otros dispositivos, como el lector de digitales, por ejemplo, puedan originar nuevos productos con funcionalidad adecuadas a los requisitos planteados. Además de la parte física, el texto también presenta la aplicación desarrollada, que viabiliza la implementación de soluciones para las necesidades presentadas. La aplicación elegida como forma de validación del proceso fue algo que toma mucho tiempo de los profesores en instituciones de enseñanza, o sea, el registro de la presencia de los alumnos. Tarea esta que demanda un tiempo razonable que podría estar siendo aplicado con más propiedad en el proceso enseñanza aprendizaje.

Palabras clave: Arduino; Biométrico; Digital; Hardware libre y control de presencia; Impresión; Reconocimiento. 